\title{
Filters and Matrix Factorization
}

\author{
Palle E. T. Jorgensen \\ Department of Mathematics, The University of Iowa, \\ Iowa City, IA52242, USA \\ jorgen@math.uiowa.edu \\ Myung-Sin Song \\ Department of Mathematics and Statistics, Southern Illinois University Edwardsville \\ Edwardsville, IL62026, USA \\ msong@siue.edu
}

\begin{abstract}
We give a number of explicit matrix-algorithms for analysis/synthesis in multi-phase filtering; i.e., the operation on discrete-time signals which allow a separation into frequency-band components, one for each of the ranges of bands, say $N$, starting with low-pass, and then corresponding filtering in the other band-ranges. If there are $N$ bands, the individual filters will be combined into a single matrix action; so a representation of the combined operation on all $N$ bands by an $N \times N$ matrix, where the corresponding matrix-entries are periodic functions; or their extensions to functions of a complex variable. Hence our setting entails a fixed $N \times N$ matrix over a prescribed algebra of functions of a complex variable. In the case of polynomial filters, the factorizations will always be finite. A novelty here is that we allow for a wide family of non-polynomial filter-banks.

Working modulo $N$ in the time domain, our approach also allows for a natural matrix-representation of both down-sampling and up-sampling. The implementation encompasses the combined operation on input, filtering, down-sampling, transmission, up-sampling, an action by dual filters, and synthesis, merges into a single matrix operation. Hence our matrixfactorizations break down the global filtering-process into elementary steps. To accomplish this, we offer a number of adapted matrix factorizationalgorithms, such that each factor in our product representation implements in a succession of steps the filtering across pairs of frequency-bands; and so it is of practical significance in implementing signal processing, including filtering of digitized images. Our matrix-factorizations are especially useful in the case of the processing a fixed, but large, number of bands.
\end{abstract}

Key words and phrases : Signals, image processing, algorithms, lifting, matrix factorization, Hilbert space, numerical methods, Fourier analysis.

2010 AMS Mathematics Subject Classification - Primary 18A32, 42C40, 46M05, 47B10, 60H05, 62M15, 65T60. 


\section{Introduction}

Two facts are recognized about multiresolutions;- one that they are versatile, and second that they offer powerful and fast algorithms. Their use in wavelet analysis, for example, takes advantage of a familiar localization property that wavelets have (by contrast to analogous Fourier tools). One is thus led to a host of recursive basis-constructions created with the use of multi-resolutions in Hilbert spaces. This philosophy carries over even to multiresolution analysis of fractals and iterated function systems in dynamics, as they too share these multi-band features. Moreover, a careful use of multiresolutions and an implied localization further yields much better pointwise approximations than is otherwise possible for traditional Fourier-bases.

In the present paper we take up a specific and algorithmic feature of this analysis: How does one effectively create an algorithm, breaking down the process into a finite number of steps, each individual step using input-output between pairs of bands in a given finite set of $N$ frequency bands. We show that this can be accomplished with a matric factorization; each matrix factor modelling the (i-j) input-output step. While a fixed polynomial signal-processing filter, corresponding to a fixed number of $N$ bands, will take the form of an $N$ by $N$ matrix having polynomial entries, the breaking down into easy implementation steps will require a relatively subtle matrix factorization; built in such a way that the matrix-factors will be alternately upper- or lower triangular, and such that the factors further will then represent local ( $\mathrm{i}-\mathrm{j}$ ) input-output operations between pairs of bands in the array of a total of $N$ bands.

While our aim is a matrix factorization covering an arbitrary number $N$ of bands, to simplify the analysis, we begin with a detailed study of the case $N=2$, and thus the 2 by 2 filter-matrix case. The key idea is more transparent there. We then proceed to the general case of $N>2$, detailed in the rest of the paper; and isolating those features which require a more subtle analysis for the case $N>2$, as compared with the $N=2$ case.

The second step in our realization of filters requires an associated representation in a suitable Hilbert spaces $\mathcal{H}$ of states; designed in such a way that specific families of closed subspaces in $\mathcal{H}$ will model "non-overlapping frequency bands." Orthogonality of these subspaces (in $\mathcal{H}$ ) then corresponds to the desirable feature of uncorrelated frequency-bands. Since the different frequency bands must exhaust the total signal for the entire system, one looks for a realization with orthogonal projections which add to the identity operator in $\mathrm{H}$. Hence this version of time/frequency analysis is non-commutative, and one is further faced with a selection of special families of commuting orthogonal projections. We show that these may be computed from the above mentioned matrix-factorization. In an operator-algebraic language, the Hilbert space framework takes the form of suitable representations of a certain non-commutative algebra of operators; 
often referred to as the Cuntz algebra, and denoted $\mathcal{O}_{N}$. The subscript $N$ corresponds to "number of frequency-bands." The algebra $\mathcal{O}_{N}$ itself is specified by its generators and relations; see sections 2 and 3 below. In general $\mathcal{O}_{N}$ has a multitude of representations in Hilbert space. Here we identify those of relevance to multi-band signal processing.

Our purpose is to establish factorization of matrices $M_{N}(\mathcal{A})$ over certain rings $\mathcal{A}$ of functions, among them the ring of polynomials, and the $L^{\infty}$ functions on the circle group $\mathbb{T}$. An equivalent formulation is the study of functions on $\mathbb{T}$ which take values in the $N \times N$ scalar matrices. The general setting is as follows: Fix $N$, and consider the group $S L_{N}(\mathcal{A})$ where the " $S$ " is for determinant $=1$. The object is then to factor arbitrary elements in $S L_{N}(\mathcal{A})$ as alternating products of upper and lower triangular matrix functions; equivalently, upper and lower triangular elements in $M_{N}(\mathcal{A})$ with the constant 1 in the diagonal.

In digital signal or image-processing one makes use of subdivisions of various families of signals into frequency bands. This is of relevance in modern-day wireless signal and image processing, and the choice of a number $N$ of frequency bands may vary from one application to the next.

There is a certain representation theoretic framework which has proved successful: one builds a representation of the basic operations on signals, filtering, down-sampling (in the complex frequency variable), up-sampling, and dual filter. These operations get represented by a system of operators in Hilbert spaces of states, say $\mathcal{H}$.

A multiresolution (see Fig. 1) then takes the form of a family of closed subspaces in $\mathcal{H}$. In this construction, "non-overlapping frequency bands" correspond to orthogonal subspaces in $\mathcal{H}$; or equivalently to systems of orthogonal projections. Since the different frequency bands must exhaust the signals for the entire system, one looks for orthogonal projections which add to the identity operator in $\mathcal{H}$. This leads to the study of certain representations of the Cuntz algebra $\mathcal{O}_{N}$, details below. Since time/frequency-analysis is non-commutative, one is further faced with a selection of special families of commuting orthogonal projections. When these iteration schemes (repeated subdivision sequences) are applied to the initial generators, one arrives at new bases and frames; and, in other applications, to wavelet families as recursive scheme.

Our study of iterated matrix-factorizations are motivated by such questions from signal processing, and arising in multi-resolution analyses. In this case, elements in the group $S L_{N}(\mathcal{A})$ of matrix-functions act on vector-functions $f$ in a complex frequency variable, where the components in $f$ correspond to a specified system of $N$ frequency-bands. When a matrix-factorization is established, then the action of the respective upper and lower triangular elements in $M_{N}(\mathcal{A})$ are especially simple, in that a lower triangular filter filters a low band, and then adds it to one of the higher bands; and similarly for the action of upper triangular matrix functions. 


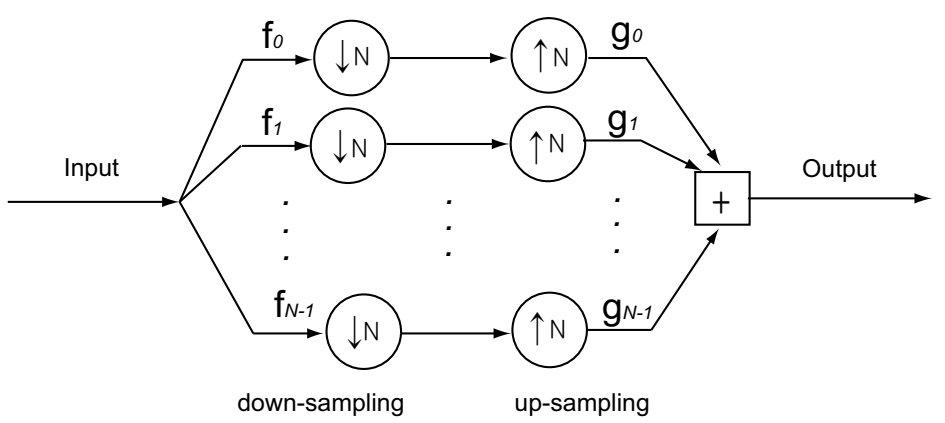

Figure 1: Perfect reconstruction in subband filtering as used in signal and image processing. Input is broken down into frequency bands, processes and then assembled (synthesis). Perfect reconstruction of output is desired.

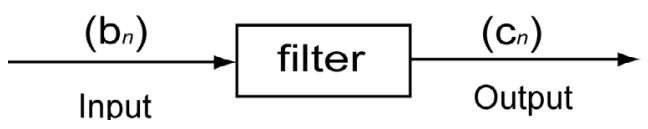

Figure 2: Filtering.

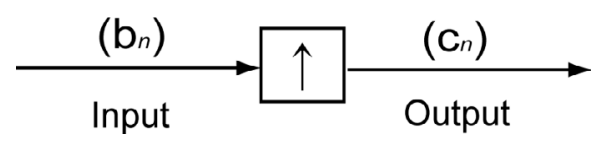

Figure 3: Upsampling.

Our analysis depend on a certain representation of the Cuntz algebra $\mathcal{O}_{N}$, where $\mathcal{O}_{N}$ is an algebra generated by the basic operations on signal representations, filtering, down-sampling (in the complex frequency variable), up-sampling, and dual filter; see Fig 1.

$$
\sum_{n} b_{n} z^{n N}=b_{0}+b_{1} z^{N}+b_{2} z^{2 N}+\cdots ;
$$

so

$$
c_{n}=\left\{\begin{array}{l}
b_{n / N} \quad \text { if } N \mid n \\
0 \quad \text { if } N \nmid n
\end{array}\right.
$$




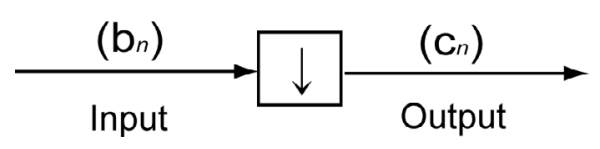

Figure 4: Downsampling.

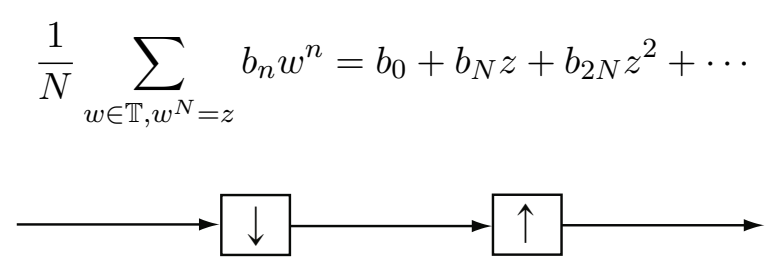

Figure 5: Downsampling and upsampling.

\section{Factorization Algorithm}

In order to illustrate our use of representations of the Cuntz algebra $O_{N}$ in algorithms for factorization, we begin with the case of $N=2$. The skeleton of these algorithms has three basic steps which we now outline.

\section{The Algorithm}

Given

$$
\left(\begin{array}{cc}
A & B \\
C & D
\end{array}\right) \in S L_{2}(\mathcal{F})
$$

where $\mathcal{F}$ is some fixed ring of functions defined on a subset $\Omega \subset \mathbb{C}$ such that $\mathbb{T} \subset \Omega$.

\section{Step 1:}

Given

$$
\mathcal{A}=\left(\begin{array}{ll}
A & B \\
C & D
\end{array}\right), \quad A D-B C \equiv 1 \quad \text { on } \mathbb{T},
$$

and set

$$
\mathcal{A}\left(z^{2}\right)\left(\begin{array}{l}
1 \\
z
\end{array}\right)=\left(\begin{array}{l}
A\left(z^{2}\right)+z B\left(z^{2}\right) \\
C\left(z^{2}\right)+z D\left(z^{2}\right)
\end{array}\right) .
$$

Let $S_{i}, i=0,1$ be

$$
\left\{\begin{array}{l}
S_{0} f(z)=f\left(z^{2}\right) \\
S_{1} f(z)=z f\left(z^{2}\right)
\end{array}\right.
$$


For the corresponding adjoint operators we therefore get:

$$
\left\{\begin{array}{l}
S_{0}^{*} f(z)=\frac{1}{2} \sum_{\omega^{2}=z} f(\omega) \\
S_{1}^{*} f(z)=\frac{1}{2} \sum_{\omega^{2}=z} \bar{\omega} f(\omega)
\end{array}\right.
$$

where the summation in (2), (3) are over points $z, \omega \in \mathbb{T}$.

Then $\left(S_{i}\right)_{i=0,1}$ are isometries in $L^{2}(\mathbb{T})$, and $S_{i}^{*} S_{j}=\delta_{i, j} I, \sum_{i=0}^{1} S_{i} S_{j}^{*}=I$ where $I$ denotes the identity operator in the Hilbert space $L^{2}(\mathbb{T})$. We will want $\mathcal{F}$ to be a ring of meromorphic functions, such that they are determined by their values on $\mathbb{T}=\{z \in \mathbb{C},|z|=1\}$; or we are simply working with functions on $\mathbb{T}$.

\section{Step 2:}

Find functions $L$ such that

$$
\left(\begin{array}{ll}
l & 0 \\
L & 1
\end{array}\right) \mathcal{A}_{\text {new }}=\mathcal{A}
$$

Solution: Apply (4) to

$$
\left(\begin{array}{l}
1 \\
z
\end{array}\right)
$$

and set

$$
\mathcal{A}_{\text {new }}\left(z^{2}\right)\left(\begin{array}{l}
1 \\
z
\end{array}\right)=\left(\begin{array}{l}
f_{0}(z) \\
f_{1}(z)
\end{array}\right)
$$

then

$$
\left\{\begin{array}{l}
f_{0}=A\left(z^{2}\right)+z B\left(z^{2}\right) \\
L\left(z^{2}\right) f_{0}(z)+f_{1}(z)=C\left(z^{2}\right)+z D\left(z^{2}\right) .
\end{array}\right.
$$

Apply $S_{i}^{*}, i=0,1$, to $(5)$

$$
\left\{\begin{array}{l}
S_{0}^{*} f_{0}=A, \quad S_{1}^{*} f_{0}=B \\
L S_{0}^{*} f_{0}+S_{0}^{*} f_{1}=C \\
L S_{1}^{*} f_{0}+S_{1}^{*} f_{1}=D . \\
\Rightarrow L=\frac{C-S_{0}^{*} f_{1}}{A} ; \quad L=\frac{D-S_{1}^{*} f_{1}}{B} .
\end{array}\right.
$$

Corollary 2.1. $A\left(S_{1}^{*} f_{1}\right)-B\left(S_{0}^{*} f_{1}\right)=1$.

Proof. Consider (6) with $\operatorname{det} \mathcal{A}=1$.

$$
\mathcal{A}=\left(\begin{array}{ll}
A & B \\
C & D
\end{array}\right)
$$

with $A D-B C=1$. So

$$
\left(\begin{array}{cc}
A & B \\
S_{0}^{*} f_{1} & S_{1}^{*} f_{1}
\end{array}\right) \in S L_{2}(\mathcal{F})
$$

We now assume $S_{i} \mathcal{F} \subset \mathcal{F}$, and $S_{i}^{*} \mathcal{F} \subset \mathcal{F}$, for all $i=0,1$. 


\section{Step 3:}

Having form $L$, from (4) we get

$$
\mathcal{A}_{\text {new }}=\left(\begin{array}{cc}
l & 0 \\
-L & 1
\end{array}\right)\left(\begin{array}{ll}
A & B \\
C & D
\end{array}\right)=\left(\begin{array}{cc}
A & B \\
-L A+C & -L B+D
\end{array}\right)=\left(\begin{array}{cc}
A & B \\
S_{0}^{*} f_{1} & S_{1}^{*} f_{1}
\end{array}\right)
$$

Step 4:

$$
\left(\begin{array}{cc}
l & U \\
0 & 1
\end{array}\right) \mathcal{A}_{\text {up }}=\mathcal{A}_{\text {new }}
$$

Set

$$
\mathcal{A}_{\text {up }}\left(z^{2}\right)\left(\begin{array}{l}
1 \\
z
\end{array}\right)=\left(\begin{array}{l}
g_{0}(z) \\
g_{1}(z)
\end{array}\right)
$$

and we get

$$
\left\{\begin{array}{l}
g_{0}(z)+U\left(z^{2}\right) g_{1}(z)=A\left(z^{2}\right)+B\left(z^{2}\right) z \\
g_{1}(z)=\left(S_{0}^{*} f_{1}\right)\left(z^{2}\right)+\left(S_{1}^{*} f_{1}\right)\left(z^{2}\right) z
\end{array} .\right.
$$

Apply $S_{i}^{*}, i=0,1$

$$
\Rightarrow\left\{\begin{array}{l}
S_{0}^{*} g_{0}+U S_{0}^{*} g_{1}=A, \quad S_{1}^{*} g_{0}+U S_{1}^{*} g_{1}=B \\
S_{0}^{*} g_{1}=S_{0}^{*} f_{1}, \quad S_{1}^{*} g_{1}=S_{1}^{*} f_{1} \\
\Rightarrow U=\frac{A-S_{0}^{*} g_{0}}{S_{0}^{*} f_{1}} \quad \text { and } \quad U=\frac{B-S_{1}^{*} g_{0}}{S_{1}^{*} f_{1}}
\end{array}\right.
$$

and continue.

$$
\begin{gathered}
S_{0}^{*} f_{0}=A, \quad S_{1}^{*} f_{0}=B \\
L S_{0}^{*} f_{0}+S_{0}^{*} f_{0}=C \\
L S_{1}^{*} f_{0}+S_{1}^{*} f_{0}=D \\
L=\frac{C-S_{0}^{*} f_{1}}{A}=\frac{D-S_{1}^{*} f_{1}}{B} \\
A\left(D-S_{1}^{*} f_{1}\right)=B\left(C-S_{0}^{*} f_{1}\right) \\
1=A S_{1}^{*} f_{1}-B S_{0}^{*} f_{1} \\
A_{\text {new }}^{(1)}=\left(\begin{array}{cc}
1 & 0 \\
-L & 1
\end{array}\right)\left(\begin{array}{cc}
A & B \\
C & D
\end{array}\right)=\left(\begin{array}{cc}
A & B \\
-L A+C & -L B+D
\end{array}\right)=\left(\begin{array}{cc}
A & B \\
S_{0}^{*} f_{1} & S_{1}^{*} f_{1}
\end{array}\right)
\end{gathered}
$$

so

$$
\left(\begin{array}{ll}
1 & 0 \\
L & 1
\end{array}\right)\left(\begin{array}{cc}
A & B \\
S_{0}^{*} f_{1} & S_{1}^{*} f_{1}
\end{array}\right)=\left(\begin{array}{cc}
A & B \\
C & D
\end{array}\right)
$$




\section{Factorization Cases}

In order to answer the main issue raised in section 1, in the present section it will be helpful to discuss briefly the group $S L_{2}$ over the ring of $L^{\infty}$ functions on $\mathbb{T}$. This discussion also serves to highlight the role of the $C^{*}$-algebra $\mathcal{O}_{2}$. The lemmas will extend from $N=2$ to $N>2$ with suitable modifications, as we outline in the subsequent section, but we find it useful to first present them in the case of $N=2$.

In the infinite-dimensional group $S L_{2}\left(L^{\infty}(\mathbb{T})\right)$, consider elements $\mathcal{A}$ with factorization as in (7):

$$
\begin{gathered}
\mathcal{A}=\left(\begin{array}{ll}
A & B \\
C & D
\end{array}\right) \\
\mathcal{A}=\left(\begin{array}{ll}
1 & 0 \\
L & 1
\end{array}\right) \mathcal{A}^{(1)}, \quad L \in L^{\infty}(\mathbb{T}), \quad \mathcal{A}^{(1)} \in S L_{2}\left(L^{\infty}(\mathbb{T})\right)
\end{gathered}
$$

that gives optimal factorization:

$$
\begin{gathered}
\mathcal{A}^{(1)}\left(z^{2}\right)\left(\begin{array}{l}
1 \\
z
\end{array}\right)=\left(\begin{array}{l}
f_{0} \\
f_{1}
\end{array}\right) \\
\left\{\begin{array}{l}
A\left(z^{2}\right)+z B\left(z^{2}\right)=f_{0}(z) \\
C\left(z^{2}\right)+z D\left(z^{2}\right)=L\left(z^{2}\right) f_{0}(z)+f_{1}(z) \quad\left\{S_{i}\right\}_{i=0} \in R E P\left(\mathcal{O}_{2}, L^{2}(\mathbb{T})\right)
\end{array}\right. \\
\left\{\begin{array}{l}
S_{0}^{*} f_{0}=A, S_{1}^{*} f_{0}=B \\
L S_{0}^{*} f_{0}+S_{0}^{*} f_{1}=C \\
L S_{1}^{*} f_{0}+S_{1}^{*} f_{1}=D
\end{array}\right. \\
\Longleftrightarrow\left\{\begin{array}{l}
S_{0}^{*} f_{1}=C-L A \\
S_{1}^{*} f_{1}=D-L B
\end{array}\right. \\
\Rightarrow f_{1}=\left(S_{0} S_{0}^{*}+S_{1} S_{1}^{*}\right) f_{1}=S_{0}(C-L A)+S_{1}(D-L B) \\
\left(\begin{array}{ll}
A & B \\
C & D
\end{array}\right) \longrightarrow\left(\begin{array}{cc}
A & B \\
S_{0}^{*} f_{1} & S_{1}^{*} f_{1}
\end{array}\right)
\end{gathered}
$$

Since $S_{i}$ is isometric for $i=1,2$.

$$
\begin{gathered}
\left\|f_{1}\right\|^{2}=\|C-L A\|^{2}+\|D-L B\|^{2} \quad \text { where }\|\cdot\| \text { is the } L^{2}(\mathbb{T})-\text { norm. } \\
\langle u, v\rangle=\int_{\mathbb{T}} \bar{u} v \quad \text { with respect to Haar measure on } \mathbb{T} \text {. }
\end{gathered}
$$

So any functions

$$
\mathcal{A}=\left(\begin{array}{ll}
1 & 0 \\
L & 1
\end{array}\right) \mathcal{A}^{(1)}
$$


we pick the one with $f_{1}$ attaching its minimum in (12)

$$
\inf \{(12) \mid \text { factorization (14) holds }\}
$$

Calculating $L$ on $\mathcal{A}$

$$
\begin{aligned}
& L_{M}(\epsilon)=L+\epsilon M, \quad M \in L^{\infty}(\mathbb{T}) . \\
& \left.\frac{d}{d \epsilon}\right|_{\epsilon=0}(12)=0 \quad \text { at a minimum. }
\end{aligned}
$$

$$
\begin{aligned}
& \Longleftrightarrow \\
& \langle M A, C-L A\rangle+\langle C-L A, M A\rangle+\langle M B, D-L B\rangle+\langle D-L B, M B\rangle \\
& =\operatorname{Re}(\langle M A, C-L A\rangle+\langle M B, D-L B\rangle)=0 \quad \forall M \in L^{\infty}(\mathbb{T}) . \\
& \quad \bar{A}(C-L A)+\bar{B}(D-L B)=0 \quad \text { pointwise a. e. on } \mathbb{T} .
\end{aligned}
$$

$\operatorname{Set} \operatorname{det} \mathcal{A}=1$,

$$
\|A\|^{2}+\|B\|^{2}>0 \quad \text { a. e. on } \mathbb{T} \text {. }
$$

So

$$
L=\frac{\bar{A} C+\bar{B} D}{|A|^{2}+|B|^{2}} \quad \text { pointwise a. e. } \mathbb{T} .
$$

Solving for matrices $\mathcal{A}^{(1)}$ in (16), we get

$$
\mathcal{A}^{(1)}=\left(\begin{array}{cc}
1 & 0 \\
-L & 1
\end{array}\right)\left(\begin{array}{ll}
A & B \\
C & D
\end{array}\right)=\left(\begin{array}{cc}
A & B \\
C-L A & D-L B
\end{array}\right) .
$$

So

$$
\mathcal{A}=\left(\begin{array}{ll}
1 & 0 \\
L & 1
\end{array}\right) \mathcal{A}^{(1)}
$$

With the above $L$ in (18) we see that

$$
\mathcal{A}=\left(\begin{array}{ll}
1 & 0 \\
L & 1
\end{array}\right) \mathcal{A}^{(1)}
$$

is the optimal factorization with a lower matrix as a left-factor.

Corollary 3.1. Given

$$
\left(\begin{array}{ll}
A & B \\
C & D
\end{array}\right) \in G L_{2}\left(L^{\infty}(\mathbb{T})\right)
$$


then the optimal solution (18) to the factorization problem

$$
\left(\begin{array}{ll}
A & B \\
C & D
\end{array}\right)=\left(\begin{array}{ll}
1 & 0 \\
L & 1
\end{array}\right)\left(\begin{array}{cc}
A & B \\
S_{0}^{*} f_{1} & S_{1}^{*} f_{1}
\end{array}\right)
$$

has the matrix

$$
\left(\begin{array}{cc}
A & B \\
S_{0}^{*} f_{1} & S_{1}^{*} f_{1}
\end{array}\right)
$$

on the right hand side in (19) orthogonal, i.e.,

$$
\bar{A}\left(S_{0}^{*} f_{1}\right)+\bar{B}\left(S_{1}^{*} f_{1}\right) \equiv 0 \quad \text { on } \mathbb{T} .
$$

Proof. When the function $L$ in (18) is used in the computation of

$$
\left(\begin{array}{cc}
A & B \\
S_{0}^{*} f_{1} & S_{1}^{*} f_{1}
\end{array}\right)
$$

we see that for any $z \in \mathbb{T},\left(\left(S_{0}^{*} f_{1}\right)(z),\left(S_{1}^{*} f_{1}\right)(z)\right)$ in $\mathbb{C}$ is in the orthogonal complement of $(A(z), B(z))$; indeed with (18) we get

$$
\begin{aligned}
& \bar{A}\left(S_{0}^{*} f_{1}\right)+\bar{B}\left(S_{1}^{*} f_{1}\right) \\
& =\bar{A}\left(C-\frac{\bar{A} C+\bar{B} D}{|A|^{2}+|B|^{2}} A\right)+\bar{B}\left(D-\frac{\bar{A} C+\bar{B} D}{|A|^{2}+|B|^{2}} B\right) \\
& =\bar{A} C+\bar{B} D-(\bar{A} C+\bar{B} D) \equiv 0 ;
\end{aligned}
$$

i.e., a pointwise identity for functions on $\mathbb{T}$.

Corollary 3.2. If $\mathcal{A} \in S U\left(L^{\infty}(\mathbb{T})\right)$ (i.e., unitary) then $L$ in (18) is 0 and so $\mathcal{A}=\mathcal{A}^{(1)}$ so the factorization steps.

Proof.

$$
\mathcal{A}=\left(\begin{array}{ll}
A & B \\
C & D
\end{array}\right)
$$

so unitary makes that the rows are orthogonal $\bar{A} C+\bar{B} D=0$ in the inner product on $\mathbb{C}^{2}$

$$
\langle z, w\rangle=\overline{z_{1}} w_{1}+\overline{z_{2}} w_{2}
$$

and $|A|^{2}+|B|^{2}=1$.

$$
\mathcal{A}^{(1)}=\left(\begin{array}{cc}
A & B \\
S_{0}^{*} f_{1} & S_{1}^{*} f_{1}
\end{array}\right)
$$

using

$$
\left(\begin{array}{ll}
S_{0}^{*} g_{0} & S_{1}^{*} g_{0} \\
S_{0}^{*} f_{1} & S_{1}^{*} f_{1}
\end{array}\right)
$$


Note this using the repeated on any $\mathcal{A}^{(1)} \in S L_{2}\left(L^{\infty}(\mathbb{T})\right)$ each time pick $L$ such that the infimum in (12) is attained.

With the same argument, we factor matrix

$$
\begin{gathered}
\left(\begin{array}{ll}
1 & U \\
0 & 1
\end{array}\right) \quad U \in L^{\infty}(\mathbb{T}) \\
\mathcal{A}=\left(\begin{array}{cc}
1 & U \\
0 & 1
\end{array}\right) \mathcal{A}^{(2)}, \quad \mathcal{A}^{(2)} \in S L_{2}\left(L^{\infty}(\mathbb{T})\right) .
\end{gathered}
$$

Set

$$
\begin{gathered}
\left(\begin{array}{l}
g_{0} \\
g_{1}
\end{array}\right)=\mathcal{A}^{(2)}\left(z^{2}\right)\left(\begin{array}{l}
1 \\
z
\end{array}\right) \\
\left\{\begin{array}{l}
A\left(z^{2}\right)+z B\left(z^{2}\right)=g_{0}+U\left(z^{2}\right) g_{1} \\
C\left(z^{2}\right)+z D\left(z^{2}\right)=g_{1}
\end{array}\right. \\
\left\{\begin{array}{l}
A=S_{0}^{*} g_{0}+U S_{0}^{*} g_{1} \\
B=S_{1}^{*} g_{0}+U S_{1}^{*} g_{1} \\
C=S_{0}^{*} g_{1}, D=S_{1}^{*} g_{1}
\end{array} \quad S_{0}^{*} g_{0}=A-U C, \quad S_{1}^{*} g_{0}=B-U D\right. \\
g_{0}=S_{0} S_{0}^{*} g_{0}+S_{1} S_{1}^{*} g_{1}=S_{0}(A-U C)+S_{1}(B-U D) \\
\left\|g_{0}\right\|^{2}=\|A-U C\|^{2}+\|B-U D\|^{2}
\end{gathered}
$$

such that (22) holds.

$$
\left(\begin{array}{ll}
A & B \\
C & D
\end{array}\right) \longrightarrow\left(\begin{array}{cc}
A-U C & B-U D \\
C & D
\end{array}\right), \quad S_{0}^{*} g_{0}=A-U C, \quad S_{1}^{*} g_{0}=B-U D
$$

Pick $U$ such that

$$
\begin{gathered}
\bar{C}(A-U C)+\bar{D}(B-U D)=0 \\
U=\frac{\bar{C} A+\bar{D} B}{|C|^{2}+|D|^{2}} \\
\mathcal{A}^{(2)}=\left(\begin{array}{cc}
S_{0}^{*} g_{0} & S_{1}^{*} g_{0} \\
C & D
\end{array}\right)
\end{gathered}
$$

in $(22)$.

If

$$
\mathcal{A}=\left(\begin{array}{ll}
A & B \\
C & D
\end{array}\right) \in S L_{2}\left(L^{\infty}(\mathbb{T})\right)
$$

then

$$
U=\frac{\bar{C} A+\bar{D} B}{|C|^{2}+|D|^{2}}=0
$$


See (26) so the factorization

$$
\mathcal{A}=\left(\begin{array}{ll}
1 & U \\
0 & 1
\end{array}\right) \mathcal{A}^{(2)}
$$

in (22) is then, $U=0 \Rightarrow \mathcal{A}=\mathcal{A}^{(2)}$. Then following factorization results:

$$
\begin{gathered}
\mathcal{A}=\left(\prod(\text { lower })(\text { upper })\right) S L_{2}\left(L^{\infty}(\mathbb{T})\right) \\
\left(\begin{array}{cc}
A & B \\
C & D
\end{array}\right) \text { factor out lower matrix on the left }\left(\begin{array}{cc}
A & B \\
S_{0}^{*} f_{1} & S_{1}^{*} f_{1}
\end{array}\right) \\
\text { factor out upper matrix on the left }\left(\begin{array}{cc}
S_{0}^{*} g_{0} & S_{1}^{*} g_{0} \\
S_{0}^{*} f_{1} & S_{1}^{*} f_{1}
\end{array}\right) .
\end{gathered}
$$

Or equivalently,

$$
\left(\begin{array}{ll}
A & B \\
C & D
\end{array}\right)=\left(\begin{array}{cc}
1 & 0 \\
L & 1
\end{array}\right)\left(\begin{array}{cc}
1 & U \\
0 & 1
\end{array}\right)\left(\begin{array}{ll}
S_{0}^{*} g_{0} & S_{1}^{*} g_{0} \\
S_{0}^{*} f_{1} & S_{1}^{*} f_{1}
\end{array}\right)
$$

Corollary 3.3. Consider $\mathcal{A} \in S L_{2}\left(L^{\infty}(\mathbb{T})\right)$, and the factorization

$$
\mathcal{A}=\left(\begin{array}{cc}
1 & 0 \\
L_{1} & 1
\end{array}\right)\left(\begin{array}{cc}
1 & U_{1} \\
0 & 1
\end{array}\right) \cdots\left(\begin{array}{cc}
1 & 0 \\
L_{p} & 1
\end{array}\right)\left(\begin{array}{cc}
1 & U_{p} \\
0 & 1
\end{array}\right)\left(\begin{array}{cc}
S_{0}^{*} g_{0} & S_{1}^{*} g_{0} \\
S_{0}^{*} f_{1} & S_{1}^{*} f_{1}
\end{array}\right)
$$

resulting from an iteration of the algorithm from (29). Then the last factor in (30) is of diagonal form if and only if the following hold: There are functions $\varphi, \psi \in L^{2}(\mathbb{T})$ such that

$$
g_{0}(z)=\varphi\left(z^{2}\right), \quad \text { and } \quad f_{1}(z)=z \psi\left(z^{2}\right)
$$

and, in this case, the last factor in (30) is as follows:

$$
\left(\begin{array}{ll}
S_{0}^{*} g_{0} & S_{1}^{*} g_{0} \\
S_{0}^{*} f_{1} & S_{1}^{*} f_{1}
\end{array}\right)=\left(\begin{array}{cc}
\varphi & 0 \\
0 & \psi
\end{array}\right)
$$

Proof. This follows from (29), and the Cuntz-relations:

$$
S_{i}^{*} S_{j}=\delta_{i, j}, \quad \sum_{i} S_{i} S_{i}^{*}=I .
$$




\subsection{Factorizations}

We fix a value of $N>1$ (i.e., the given number of frequency bands), and we begin with the formula for a canonical system of $N$ isometries $S_{i}$ which define an associated representation of the Cuntz algebra $O_{N}$. Said differently: The system of isometries $\left\{S_{i}\right\}$ satisfies the Cuntz relations with reference to the Hilbert space $L^{2}(\mathbb{T})$ where $\mathbb{T}$ is the circle group (one-torus) with its normalized invariant Haar measure. When the value of $N$ is fixed, then the multi-resolution filters will then take the form of $N \times N$ matrix functions; the matrix entries might be polynomials, or, more generally, functions from $L^{\infty}(\mathbb{T})$. Hence the questions about matrix factorization depends on the context. In the case of polynomial entries we will make use of degree, but this is not available for the more general case of entries from the algebra $L^{\infty}(\mathbb{T})$. In every one of the settings, we develop factorization algorithms, and the particular representation of the Cuntz algebra will play an important role.

Let the set of all orthogonal $N$-filters be denoted $\mathcal{O F}_{N}[16]$. The standard representation of $O_{N}$, which we will use below, is given by the system of isometries $\left\{S_{j}\right\}$ as follows:

$$
\left(S_{j} \varphi\right)(z)=f_{j}(z) \varphi\left(z^{N}\right) .
$$

Lemma 3.4. [16] Let $N \in \mathbb{Z}_{+}$be given and let $F=\left(f_{j}\right)_{j \in \mathbb{Z}_{+}}$be a function system. Then $F \in \mathcal{O F}_{N}$ if and only if the operators $S_{j}$ (34) satisfy

$$
\begin{gathered}
S_{j}^{*} S_{k}=\delta_{j, k} I \\
\sum_{j \in \mathbb{Z}_{N}} S_{j} S_{j}^{*}=I,
\end{gathered}
$$

where $I$ denotes the identity operator in $\mathcal{H}=L^{2}(\mathbb{T})$.

We say that the isometries $\left\{S_{j}\right\}_{j \in \mathbb{Z}_{N}}$ define a representation of the Cuntzalgebra $\mathcal{O}_{N},\left(S_{j}\right) \in \operatorname{Rep}\left(\mathcal{O}_{N}, L^{2}(\mathbb{T})\right)$.

Let $\mathcal{F}_{2}(N):=L^{2}\left(\mathbb{T}, \mathbb{C}^{N}\right)=\sum_{0}^{N-1} \oplus L^{2}(\mathbb{T})$ where the notation in the summation symbol means orthogonal direct sum with

$$
\|F\|_{2}^{2}=\sum_{j=0}^{N-1}\left\|f_{j}\right\|_{L^{2}(\mathbb{T})}^{2}<\infty .
$$

We will be making use of the special vector $b \in \mathcal{F}_{2}(N)$,

$$
b(z)=\left[\begin{array}{c}
1 \\
z \\
z^{2} \\
\vdots \\
z^{N-1}
\end{array}\right]
$$




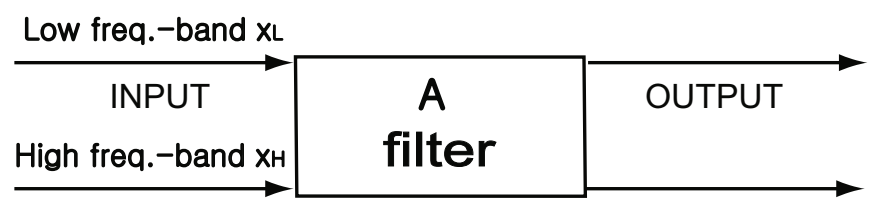

Figure 6: Filters.

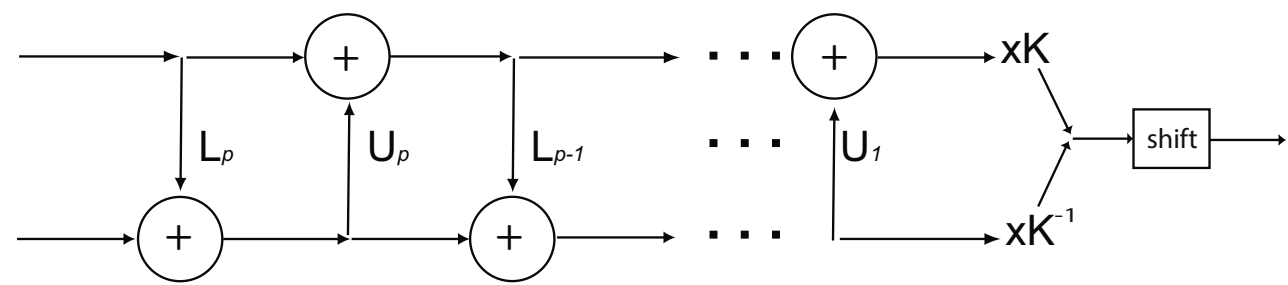

see $[16]$.

Lemma 3.5. [16] Let $N \in \mathbb{Z}_{+}$be fixed, $N>1$, and let $A=\left(A_{j, k}\right)$ be an $N \times N$ matrix-function with $A_{j, k} \in L^{2}(\mathbb{T})$. Then the following two conditions are equivalent:

(i) For $F=\left(f_{j}\right) \in \mathcal{F}_{2}(N)$, we have $F(z)=A\left(z^{N}\right) b(z)$.

(ii) $A_{i, j}=S_{j}^{*} f_{i}$ where the operators $S_{i}$ are from the Cuntz-relations $(35,36)$.

See [16] for proof.

Theorem 3.6. (Sweldens [25], [16]) Let $A \in S L_{2}$ (pol), then there are l, $p \in \mathbb{Z}_{+}$, $K \in \mathbb{C} \backslash\{0\}$ and polynomial functions $U_{1}, \ldots, U_{p}, L_{1}, \ldots, L_{p}$ such that

$$
A(z)=z^{l}\left(\begin{array}{cc}
K & 0 \\
0 & K^{-1}
\end{array}\right)\left(\begin{array}{cc}
1 & U_{1}(z) \\
0 & 1
\end{array}\right)\left(\begin{array}{cc}
1 & 0 \\
L_{1}(z) & 1
\end{array}\right) \cdots\left(\begin{array}{cc}
1 & U_{p}(z) \\
0 & 1
\end{array}\right)\left(\begin{array}{cc}
1 & 0 \\
L_{p}(z) & 1
\end{array}\right) .
$$

The filter algorithm corresponding to the matrix-factorization in (37) is as follows: And in steps:

Remark 3.7. [16] Note that if

$$
\left(\begin{array}{ll}
\alpha & \beta \\
\gamma & \delta
\end{array}\right) \in S L_{2}(\mathrm{pol})
$$

then one of the two functions $\alpha(z)$ or $\delta(z)$ must be a monomial. 


\subsection{The $2 \times 2$ case: Polynomials}

From [16], to highlight the general ideas, we begin with some details worked out in the $2 \times 2$ case; see equation (28).

To get finite algorithms, we should assume in the present subsection that the matrix-entries are polynomials.

First note that from the setting in Theorem 3.6, we may assume that matrix entries have the form $f_{H}(z)$ but with $H \subset\{0,1,2, \cdots\}$, i.e., $f_{H}(z)=a_{0}+a_{1} z+$ $\cdots$. This facilitates our use of the Euclidean algorithm.

Specifically, if $f$ and $g$ are polynomials (i.e., $H \subset\{0,1,2, \cdots\}$ ) and if $\operatorname{deg}(g) \leq \operatorname{deg}(f)$, the Euclidean algorithm yields

$$
f(z)=g(z) q(z)+r(z)
$$

with $\operatorname{deg}(r)<\operatorname{deg}(g)$. We shall write

$$
q=q u o t(g, f), \quad \text { and } \quad r=\operatorname{rem}(g, f) .
$$

Since

$$
\left(\begin{array}{cc}
K & 0 \\
0 & K^{-1}
\end{array}\right)\left(\begin{array}{cc}
1 & U \\
0 & 1
\end{array}\right)=\left(\begin{array}{cc}
1 & K^{2} U \\
0 & 1
\end{array}\right)\left(\begin{array}{cc}
K & 0 \\
0 & K^{-1}
\end{array}\right)
$$

we may assume that the factor

$$
\left(\begin{array}{cc}
K & 0 \\
0 & K^{-1}
\end{array}\right)
$$

from the equation (40) factorization occurs on the rightmost place.

$$
F=U_{N}[b],
$$

where $U$ is a unitary matrix-function, where

$$
b=\left(\begin{array}{c}
1 \\
z \\
z^{2} \\
\vdots \\
z^{N-1}
\end{array}\right)
$$

and where $U_{N}[b](z)=U\left(z^{N}\right) b(z)$.

Let $U$ represent scalar valued matrix entry in a matrix function. We now proceed to determine the polynomials $U_{1}(z), L_{1}(z), \cdots$, etc. inductively starting with

$$
A=\left(\begin{array}{ll}
1 & U \\
0 & 1
\end{array}\right) B,
$$


where $U$ and $B$ are to be determined. Introducing 41), this reads

$$
A\left(z^{2}\right)\left(\begin{array}{l}
1 \\
z
\end{array}\right)=\left(\begin{array}{cc}
1 & U\left(z^{2}\right) \\
0 & 1
\end{array}\right) B\left(z^{2}\right)\left(\begin{array}{l}
1 \\
z
\end{array}\right)=\left(\begin{array}{cc}
1 & U\left(z^{2}\right) \\
0 & 1
\end{array}\right)\left(\begin{array}{l}
h(z) \\
k(z)
\end{array}\right) .
$$

But the matrix function

$$
A=\left(\begin{array}{ll}
\alpha & \beta \\
\gamma & \delta
\end{array}\right)
$$

is given and fixed see Remark 3.7. Hence

$$
\gamma\left(z^{2}\right)+\delta\left(z^{2}\right) z=k(z)
$$

is also fixed. The two polynomials to be determined are $U$ and $h$ in (42). Carrying out the matrix product in (42) yields:

$$
\alpha\left(z^{2}\right)+\beta\left(z^{2}\right) z=h(z)+U\left(z^{2}\right) k(z)=h_{0}(z)+h_{1}\left(z^{2}\right) z+U\left(z^{2}\right)\left\{\gamma\left(z^{2}\right)+\delta\left(z^{2}\right) z\right\}
$$

where we used the orthogonal splitting

$$
L^{2}(\mathbb{T})=S_{0} S_{0}^{*} L^{2}(\mathbb{T}) \oplus S_{1} S_{1}^{*} L^{2}(\mathbb{T})
$$

from Lemma 3.4. Similarly, from (43), we get

$$
\gamma\left(z^{2}\right)+\delta\left(z^{2}\right) z=k_{0}\left(z^{2}\right)+k_{1}\left(z^{2}\right) z
$$

and therefore $\gamma=k_{0}$ and $\delta=k_{1}$, by Lemma 3.5.

Collecting terms and using the orthogonal splitting (44) we arrive at the following system of polynomial equations:

$$
\left\{\begin{array}{l}
\alpha=h_{0}+U \gamma \\
\beta=h_{1}+U \delta
\end{array}\right.
$$

or more precisely,

$$
\left\{\begin{array}{l}
\alpha(z)=h_{0}(z)+U(z) \gamma(z) \\
\beta(z)=h_{1}(z)+U(z) \delta(z) .
\end{array}\right.
$$

It follows that the two functions $U$ and $h$ may be determined from the Euclidean algorithm. With (40), we get

$$
\left\{\begin{array}{l}
U=q u o t(\gamma, \alpha) \\
h_{0}=\operatorname{rem}(\gamma, \alpha) \\
h_{1}=\operatorname{rem}(\delta, \beta) .
\end{array}\right.
$$


Remark 3.8. [16] The relevance of the determinant condition we have from Theorem 3.6 is as follows:

$$
\operatorname{det} A=\alpha \delta-\beta \gamma \equiv 1 .
$$

Substitution of (45) into this yields:

$$
h_{0} \delta-h_{1} \gamma \equiv 1 .
$$

Solutions to (45) are possible because the two polynomials $\delta(z)$ and $\gamma(z)$ are mutually prime. The derived matrix

$$
\left(\begin{array}{cc}
h_{0} & h_{1} \\
\gamma & \delta
\end{array}\right)
$$

is obtained from $A$ via a row-operation in the ring of polynomials.

For the inductive step, it is important to note:

$$
\operatorname{deg}\left(h_{0}\right)<\operatorname{deg}(\gamma), \quad \text { and } \operatorname{deg}\left(h_{1}\right)<\operatorname{deg}(\delta) .
$$

The next step, continuing from (42) is the determination of a matrix-function $C$ and three polynomials $p, q$, and $L$ such that

$$
\left(\begin{array}{cc}
1 & -U \\
0 & 1
\end{array}\right) A=\left(\begin{array}{ll}
1 & 0 \\
L & 1
\end{array}\right) C
$$

and

$$
\left(\begin{array}{cc}
1 & -U\left(z^{2}\right) \\
0 & 1
\end{array}\right) A\left(z^{2}\right)\left(\begin{array}{l}
1 \\
z
\end{array}\right)=\left(\begin{array}{cc}
1 & 0 \\
L\left(z^{2}\right) & 1
\end{array}\right)\left(\begin{array}{l}
p(z) \\
q(z)
\end{array}\right)
$$

Here

$$
\left(\begin{array}{l}
p \\
q
\end{array}\right)=C\left(z^{2}\right)\left(\begin{array}{l}
1 \\
z
\end{array}\right)
$$

The reader will notice that in this step, everything is as before with the only difference that now

$$
\left(\begin{array}{ll}
1 & 0 \\
L & 1
\end{array}\right)
$$

is lower diagonal in contrast with

$$
\left(\begin{array}{ll}
1 & U \\
0 & 1
\end{array}\right)
$$

in the previous step.

This time, the determination of the polynomial $p$ in (49) is automatic. With

$$
p(z)=p_{0}\left(z^{2}\right)+z p_{1}\left(z^{2}\right)
$$


(see (44)) and we get the following system:

$$
\begin{gathered}
\left\{\begin{array}{l}
p_{0}=\alpha-U \gamma=h_{0} \\
p_{1}=\beta-U \delta=h_{1} ; \quad \text { and }
\end{array}\right. \\
\left\{\begin{array}{l}
\gamma=L(\alpha-U \gamma)+q_{0}=L h_{0}+q_{0} \\
\delta=L(\beta-U \delta)+q_{1}=L h_{1}+q_{1}
\end{array}\right.
\end{gathered}
$$

So the determination of $L(z)$ and $q(z)=q_{0}\left(z^{2}\right)+z q_{1}\left(z^{2}\right)$ may be done with Euclid:

$$
\left\{\begin{array}{l}
L=q u o t(\alpha-U \gamma, \gamma)=q u o t\left(h_{0}, \gamma\right) \\
q_{0}=\operatorname{rem}(\alpha-U \gamma, \gamma)=\operatorname{rem}\left(h_{0}, \gamma\right) \\
q_{1}=\operatorname{rem}(\beta-U \delta, \delta)=\operatorname{rem}\left(h_{1}, \delta\right)
\end{array}\right.
$$

Combining the two steps, the comparison of degrees is as follows:

$$
\left\{\begin{array}{l}
\operatorname{deg}\left(q_{0}\right)<\operatorname{deg}\left(h_{0}\right)<\operatorname{deg}(\gamma) \\
\operatorname{deg}\left(q_{1}\right)<\operatorname{deg}\left(h_{1}\right)<\operatorname{deg}(\delta)
\end{array} .\right.
$$

Two conclusions now follow:

(i) the procedure may continure by recursion;

(ii) the procedure must terminate.

Remark 3.9. In order to start the algorithm in (46) with direct reference to Euclid, we must have

$$
\operatorname{deg}(\gamma) \leq \operatorname{deg}(\alpha)
$$

where

$$
A=\left(\begin{array}{ll}
\alpha & \beta \\
\gamma & \delta
\end{array}\right)
$$

is the initial $2 \times 2$ matrix-function.

Now, suppose (52), i.e., that

$$
\operatorname{deg}(\gamma) \leq \operatorname{deg}(\alpha)
$$

Then determine a polynomial $L$ such that

$$
\operatorname{deg}(\gamma-L \alpha) \leq \operatorname{deg}(\alpha)
$$

We may then start the procedure (46) on the matrix function

$$
\left(\begin{array}{cc}
\alpha & \beta \\
\gamma-L \alpha & \delta
\end{array}\right)=\left(\begin{array}{cc}
1 & 0 \\
-L & 1
\end{array}\right) A
$$


If a polynomial $U$ and a matrix function $B$ is then found for

$$
\left(\begin{array}{cc}
\alpha & \beta \\
\gamma-L \alpha & \delta
\end{array}\right)
$$

then the factorization

$$
A=\left(\begin{array}{ll}
1 & 0 \\
L & 1
\end{array}\right)\left(\begin{array}{ll}
1 & U \\
0 & 1
\end{array}\right) B
$$

holds; and the recursion will then work as outlined.

In the following, starting with a matrix-function $A$, we will always assume that the degrees of the polynomials $\left(A_{i, j}\right)_{i, j \in \mathbb{Z}_{N}}$ have been adjusted this way, so the direct Euclidean algorithm can be applied.

\subsection{The $3 \times 3$ case}

The thrust of this section is the assertion that Theorem 3.6 holds with small modifications in the $3 \times 3$ case.

\subsubsection{Comments:}

In the definition of $A \in S L_{3}(\mathrm{pol})$, it is understood that $A(z)$ has $\operatorname{det} A(z) \equiv 1$ and that the entries of the inverse matrix $A(z)^{-1}$ are again polynomials.

Note that if $L, M, U$ and $V$ are polynomials, then the four matrices

$$
\left(\begin{array}{ccc}
1 & 0 & 0 \\
L & 1 & 0 \\
0 & M & 1
\end{array}\right),\left(\begin{array}{ccc}
1 & 0 & 0 \\
0 & 1 & 0 \\
L & 0 & 1
\end{array}\right),\left(\begin{array}{ccc}
1 & U & 0 \\
0 & 1 & V \\
0 & 0 & 1
\end{array}\right) \text { and }\left(\begin{array}{ccc}
1 & 0 & U \\
0 & 1 & 0 \\
0 & 0 & 1
\end{array}\right)
$$

are in $S L_{3}(\mathrm{pol})$ since

$$
\begin{gathered}
\left(\begin{array}{ccc}
1 & 0 & 0 \\
L & 1 & 0 \\
0 & M & 1
\end{array}\right)^{-1}=\left(\begin{array}{ccc}
1 & 0 & 0 \\
-L & 1 & 0 \\
L M & -M & 1
\end{array}\right) \text { and } \\
\left(\begin{array}{ccc}
1 & U & 0 \\
0 & 1 & V \\
0 & 0 & 1
\end{array}\right)^{-1}=\left(\begin{array}{ccc}
1 & -U & U V \\
0 & 1 & -V \\
0 & 0 & 1
\end{array}\right)
\end{gathered}
$$

Theorem 3.10. [16] Let $A \in S L_{3}(\mathrm{pol})$; then the conclusion in Theorem 3.6 carries over with the modification that the alternating upper and lower triangular matrix-functions now have the form (54) or (55)-(56) where the functions $L_{j}, M_{j}, U_{j}$ and $V_{j}, j=1,2, \cdots$ are polynomials. 


\subsection{The $N \times N$ case}

Below we outline the modifications to our algorithms from the $2 \times 2$ case needed in order to deal with filters with $N(>2)$ bands, hence factorization of $N \times N$ matrix functions. The main difference when the number of frequency bands $N$ is more than 2 is that in our factorizations, both the lower and the upper triangular factors, must take into account operations which cross between any pair of the total system of $N$ frequency bands.

Theorem 3.11. [16] Let $N \in \mathbb{Z}_{+}, N>1$, be given and fixed. Let $A \in S L_{N}($ pol); then the conclusions in Theorem 3.6 carry over with the modification that the alternative factors in the product are upper and lower triangular matrix-functions in $S L_{N}($ pol $)$. We may take the lower triangular matrix-factors $\mathcal{L}=\left(L_{i, j}\right)_{i, j \in \mathbb{Z}_{N}}$ of the form

$$
\left(\begin{array}{cccccccc}
1 & 0 & 0 & 0 & 0 & 0 & 0 & 0 \\
0 & 1 & 0 & 0 & 0 & 0 & 0 & 0 \\
L_{p} & 0 & 1 & 0 & 0 & 0 & 0 & 0 \\
0 & L_{p+1} & 0 & 1 & 0 & 0 & 0 & 0 \\
0 & 0 & . & 0 & 1 & 0 & 0 & 0 \\
0 & 0 & 0 & . & 0 & 1 & 0 & 0 \\
0 & 0 & 0 & 0 & . & 0 & 1 & 0 \\
0 & 0 & 0 & 0 & 0 & L_{N-1} & 0 & 1
\end{array}\right)
$$

polynomial entries

$$
\left\{\begin{array}{l}
L_{i, i} \equiv 1, \\
L_{i, j}(z)=\delta_{i-j, p} L_{i}(z)
\end{array}\right.
$$

and the upper triangular factors of the form $\mathcal{U}=\left(U_{i, j}\right)_{i, j \in \mathbb{Z}_{N}}$ with

$$
\left\{\begin{array}{l}
U_{i, i} \equiv 1, \\
L_{i, j}(z)=\delta_{i-j, p} U_{i}(z) .
\end{array}\right.
$$

Proof. Notation. Let $U_{1}, \cdots, U_{N}, L_{1}, \cdots, L_{N}$ be polynomials and set

$$
\mathcal{U}_{N}(U)=\left(\begin{array}{ccccccc}
1 & U_{1} & 0 & 0 & 0 & 0 & 0 \\
0 & 1 & U_{2} & 0 & 0 & 0 & 0 \\
0 & 0 & 1 & . & 0 & 0 & 0 \\
0 & 0 & 0 & 1 & . & 0 & 0 \\
0 & 0 & 0 & 0 & 1 & . & 0 \\
0 & 0 & 0 & 0 & 0 & 1 & U_{N-1} \\
0 & 0 & 0 & 0 & 0 & 0 & 1
\end{array}\right)
$$




$$
\mathcal{L}_{N}(L)=\left(\begin{array}{ccccccc}
1 & 0 & 0 & 0 & 0 & 0 & 0 \\
L_{1} & 1 & 0 & 0 & 0 & 0 & 0 \\
0 & L_{2} & 1 & 0 & 0 & 0 & 0 \\
0 & 0 & . & 1 & 0 & 0 & 0 \\
0 & 0 & 0 & . & 1 & 0 & 0 \\
0 & 0 & 0 & 0 & . & 1 & 0 \\
0 & 0 & 0 & 0 & 0 & L_{N-1} & 1
\end{array}\right)
$$

Note that both are in $S L_{N}(\mathrm{pol})$; and we have

$$
\begin{gathered}
\mathcal{U}_{N}(U)^{-1}=\mathcal{U}_{N}(-U) \quad \text { and } \\
\mathcal{L}_{N}(L)^{-1}=\mathcal{L}_{N}(-L) .
\end{gathered}
$$

Step 1: Starting with $A=\left(A_{i, j}\right) \in S L_{N}$ (pol). Then left-multiply with a suitably chosen $\mathcal{U}_{N}(-U)$ such that the degrees in the first column of $\mathcal{U}_{N}(-U) A$ decrease, i.e.,

$$
\operatorname{deg}\left(A_{0,0}\right) \leq \operatorname{deg}\left(A_{1,0}-u_{2} A_{1,0}\right) \leq \cdots \operatorname{deg}\left(A_{N-1,0}\right) .
$$

In the following, we shall use the same letter $A$ for the modified matrix-function.

Step 2: Determine a system of polynomials $L_{1}, \cdots, L_{N-1}$ and a polynomial vector-function

$$
\left[\begin{array}{c}
f_{0} \\
f_{1} \\
\cdots \\
f_{N-1}
\end{array}\right]
$$

such that

$$
A^{N}\left[\begin{array}{c}
1 \\
z \\
z^{2} \\
\cdots \\
z^{N-1}
\end{array}\right]=\mathcal{L}_{N}(L)\left[\begin{array}{c}
f_{0} \\
f_{1} \\
\cdots \\
f_{N-1}
\end{array}\right]
$$

or equivalently

$$
\sum_{j=0}^{N-1} A_{i, j}\left(z^{N}\right) z^{j}=\left\{\begin{array}{ll}
f_{0}(z) & \text { if } i=0 \\
L_{i}\left(z^{N}\right) f_{i-1}(z)+f_{i}(z) & \text { if } i>0
\end{array} .\right.
$$

Step 3: Apply the operators $S_{j}$ and $S_{j}^{*}$ from section 3 to both sides in (62). First (62) takes the form:

$$
\sum_{j=0}^{N-1} S_{j} A_{i, j}=\left\{\begin{array}{ll}
f_{0} & \text { if } i=0 \\
S_{f_{i-1}} L_{i}+f_{i} & \text { if } i>0
\end{array} .\right.
$$


For $i=1$, we get

$$
A_{1, j}=L_{1} A_{0, j}+k_{j} \quad \text { where } \quad k_{j}=S_{j}^{*} f_{1} .
$$

By (61) and the assumptions on the matrix-functions, we note that the system (63) may now be solved with the Euclidean algorithm:

$$
\left\{\begin{array}{l}
L_{1}=\operatorname{quot}\left(A_{0, j}, A_{1, j}\right) \\
k_{j}=\operatorname{rem}\left(A_{0, j}, A_{1, j}\right)
\end{array}\right.
$$

with the same polynomial $L_{1}$ for $j=0,1, \cdots, N-1$.

For the polynomial function $f_{1}$ we then have

$$
f_{1}=\sum_{j=0}^{N-1} S_{j} k_{j} ;
$$

i.e.

$$
f_{1}(z)=k_{0}\left(z^{N}\right)+k_{1}\left(z^{N}\right) z+\cdots+k_{N-1}\left(z^{N-1}\right) z^{N-1} .
$$

The process now continues recursively until all the functions $L_{1}, L_{2}, \cdots, f_{1}, f_{2}, \cdots$ have been determined.

Step 4: The formula (62) translates into a matrix-factorizations as follows: With $L$ and $F$ determined in (62), we get

$$
A=\mathcal{L}_{N}(L) B
$$

as a simple matrix-product taking $B=\left(B_{i, j}\right)$ and

$$
B_{i, j}=S_{j}^{*} f_{i}
$$

where we used Lemmas 3.4 and 3.5.

Step 5: The process now continues with the polynomial matrix-function from (66) and (67). We determine polynomials $U_{1}, \cdots, U_{N-1}$ and a third matrix function

$$
C=(C(z))=\left(C_{i, j}(z)\right) \text { such that } B=\mathcal{U}_{N}(U) C .
$$

Step 6: As each step of the process we alternate $L$ and $U$; and at each step, the degrees of the matrix-functions is decreased. Hence the recursion must terminate as stated in Theorem 3.11.

\section{$3.5 \quad L^{\infty}(\mathbb{T})$-matrix entries.}

While the case $N=2$ is motivated by application to the high-pass v.s. low-pass filters, may result for the $N>2$ case carry over. To see this, we first define the Cuntz-algebra $\mathcal{O}_{N}$ in general the relations are

$$
S_{i}^{*} S_{j}=\delta_{i, j} I, \quad \sum_{i} S_{i} S_{i}^{*}=I
$$


when the elements $\left(S_{i}\right)_{i=0}^{N-1}$ are given to be symmetric.

Each case (68) has many representations; for example if $\left(m_{i}(z)\right)_{i=0}^{N-1}, z \in \mathbb{T}$, is a system of filters corresponding to $N$ frequency bands, we may obtain a representation of $\mathcal{O}_{N}$ acting on the Hilbert space $L^{2}(\mathbb{T})$ as follows

$$
\left(S_{i} \psi\right)(z)=m_{i}(z) \psi\left(z^{N}\right), \quad \forall z \in \mathbb{T}, \quad \psi \in L^{2}(\mathbb{T}) .
$$

For $i \in\{0,1, \cdots, N-1\}$, the adjoint operator of $S_{i}$ in $(69)$ is

$$
\left(S_{i}^{*} \psi\right)(z)=\frac{1}{N} \sum_{w^{N}=z} \overline{m_{i}(z)} \psi\left(z^{N}\right), \quad z \in \mathbb{T} .
$$

A direct verification shows that the Cuntz-relation (68) are satisfied for the operators $\left(S_{i}\right)_{i=0}^{N-1}$ in $(69)$ if and only if the system $\left(m_{i}\right)_{i=0}^{N-1}$ is a multi-band filter covering the $N$ frequency bands.

The simplest example of the representation in (69) is the case where $m_{i}(z)=$ $z^{i}, i=0,1, \cdots, N-1$; and so

$$
\left(S_{i} \psi\right)(z)=z^{i} \psi\left(z^{N}\right), \quad i=0,1, \cdots, N-1, \quad z \in \mathbb{T}, \quad \psi \in L^{2}(\mathbb{T}) .
$$

Theorem 3.12. Let $g=\left(g_{i j}\right)_{i, j=0}^{N-1} \in S L_{N}\left(L^{\infty}(\mathbb{T})\right.$, i.e., $\left.g_{i j}(\cdot) \in L^{\infty}(\mathbb{T})\right)$, and

$$
\operatorname{detg}(\cdot) \equiv 1 \quad \text { on } \mathbb{T}
$$

then for every factorization

$$
g(z)=\left(\begin{array}{ccccc}
1 & 0 & 0 & \cdots & 0 \\
L_{1}(z) & 1 & 0 & \cdots & 0 \\
L_{2}(z) & 0 & 1 & \ddots & 0 \\
\vdots & \vdots & \vdots & \ddots & \vdots \\
\vdots & \vdots & \vdots & \ddots & \vdots \\
L_{N-1}(z) & 0 & \cdots & 0 & 1
\end{array}\right) g^{(n e w)}(z) \quad \text { (matrix product) }
$$

there is a unique $f_{i} \in L^{\infty}(\mathbb{T})$ such that

$$
\begin{gathered}
g_{0, j}^{(\text {new })}(z)=g_{0, j}(z), \quad \text { and } \\
g_{i, j}^{(\text {new })}(z)=S_{j}^{*} f_{i}, \quad \text { for } i=1,2, \cdots, N-1
\end{gathered}
$$

where $\left\{S_{j}\right\}_{j=0}^{N-1}$ is the system of Cuntz-isometries from (71). 
Proof. With the arguments above, in the space $\mathcal{O}_{N}$ of $N=2$, we now get matrix, the system:

$$
\begin{gathered}
g^{(\text {new })}\left(z^{N}\right)\left(\begin{array}{c}
1 \\
z \\
z^{2} \\
\vdots \\
z^{N-1}
\end{array}\right)=\left(\begin{array}{c}
f_{0}(z) \\
f_{1}(z) \\
f_{2}(z) \\
\vdots \\
f_{N-1}(z)
\end{array}\right), \\
\left\{\begin{array}{l}
S_{j}^{*} f_{0}=g_{0, j}, \\
L_{i} g_{0, j}+S_{j}^{*} f_{i}=g_{i, j},
\end{array}\right.
\end{gathered}
$$

and

$$
f_{i}=\sum_{j=0}^{N-1} S_{j} S_{j}^{*} f_{i}=\sum_{j=0}^{N-1} S_{j}\left(g_{i, j}-L_{i} g_{0, j}\right)
$$

for $i=1,2, \cdots, N-1$, which is desired conclusion.

\subsection{Optimal factorization in the case of $S L_{N}\left(L^{\infty}(\mathbb{T})\right)$}

Fix $N>2$, and consider the usual inner product in $\mathbb{C}^{N}$,

$$
\langle z, w\rangle:=\sum_{j=0}^{N-1} \overline{z_{j}} w_{j}
$$

defined for all $z=\left(z_{0}, \cdots, z_{N-1}\right)$, and $w=\left(w_{0}, \cdots, w_{N-1}\right)$.

For $g=\left(g_{i j}(z)\right)_{i, j=0}^{N-1} \in S L_{N}\left(L^{\infty}(\mathbb{T})\right)$, set

$$
\tilde{g_{0}}(z)=\left(g_{0 j}(z)\right)_{j=0}^{N-1}, \quad \text { a.e. },
$$

the first row in the matrix-function $\mathbb{T} \ni Z \mapsto(g(z)) \in S L_{N}\left(L^{\infty}(\mathbb{T})\right)$. Let $P(z)=P^{(g)}(z)$ denote the projection of $\mathbb{C}^{N}$ onto the one-dimensional subspace generated by $\tilde{g_{0}}(z) \in \mathbb{C}^{N}$.

Note that $(P(z))_{z \in \mathbb{T}}$ is a field of orthogonal rank-2 projection in $\mathbb{C}^{N}$. Setting

$$
\left\|\tilde{g_{0}}(z)\right\|_{2}^{2}=\sum_{j=0}^{N-1}\left|g_{0, j}(z)\right|^{2}
$$

we have:

$$
P(z) \xi=\sum_{j=0}^{N-1} \frac{\overline{g_{0, j}(z)} \xi_{j}}{\left\|\tilde{g_{0}}(z)\right\|_{2}^{2}} g_{0, j}(z) \quad \text { for all } \xi=\left(\xi 0, \cdots, \xi_{N-1}\right) \in \mathbb{C}^{N}
$$

and set

$$
{\tilde{g_{j}}}^{(n e w)}(z)=\tilde{g_{0}}(z)-P(z) \tilde{g_{j}}(z) .
$$


Corollary 3.13. (i) For the factorization (73) in Theorem 3.12, the optimal choice is that given by the matrix-factor $f^{(n e w)}$ having as rows the vector fields $\tilde{g}_{i}{ }^{(n e w)}(z)$ specified in (82).

(ii) With the resolution of row-vector fields,

$$
{\tilde{g_{j}}}^{(n e w)}(z)=\left(S_{0}^{*} f_{i}, S_{1}^{*} f_{i}, \cdots, S_{N-1}^{*} f_{i}\right)
$$

from (75), the optimal solution is attained; and it is the unique minimizer for the following system of optimization problems:

$$
\min _{f_{i} \in L^{2}(\mathbb{T})}\left\|f_{i}\right\|_{L^{2}(\mathbb{T}}^{2}, \quad 1 \leq i<N
$$

where each choice $\left(f_{i}\right)_{i=1}^{N-1}$ yields a matrix-factor $\mathcal{A}^{(\text {new })}$ via (83).

Proof. The proof of the conclusions in (i)-(ii) in the corollary follows from the arguments in the proof of Theorem 3.12 above.

\section{ACKNOWLEDGEMENTS}

We wish to thank the referees for their care in reviewing our paper, and making constructive suggestions for improvements. They are followed and they are much appreciated.

\section{References}

[1] Fatma Abdelkefi. Performance of sigma-delta quantizations in finite frames. IEEE Trans. Inform. Theory, 54(11):5087-5101, 2008.

[2] N. I. Akhiezer. The classical moment problem and some related questions in analysis. Translated by N. Kemmer. Hafner Publishing Co., New York, 1965 .

[3] W. R. Bennett. Spectra of quantized signals. Bell System Tech. J., 27:446$472,1948$.

[4] Ola Bratteli and Palle Jorgensen. Wavelets through a looking glass. Applied and Numerical Harmonic Analysis. Birkhäuser Boston Inc., Boston, MA, 2002. The world of the spectrum.

[5] Ola Bratteli and Palle E. T. Jorgensen. Wavelet filters and infinitedimensional unitary groups. In Wavelet analysis and applications (Guangzhou, 1999), volume 25 of AMS/IP Stud. Adv. Math., pages 3565. Amer. Math. Soc., Providence, RI, 2002. 
[6] John J. Benedetto, Onur Oktay, and Aram Tangboondouangjit. Complex sigma-delta quantization algorithms for finite frames. In Radon transforms, geometry, and wavelets, volume 464 of Contemp. Math., pages 27-49. Amer. Math. Soc., Providence, RI, 2008.

[7] Chris Brislawn "Group Lifting Structures for Multirate Filter Banks I: Uniqueness of Lifting Factorizations," IEEE Transactions on Signal Processing, 58(4):2068-2077, 2010.

[8] Chris Brislawn "Group Lifting Structures for Multirate Filter Banks II: Linear Phase Filter Banks," IEEE Transactions on Signal Processing, 58(4):2078-2087, 2010.

[9] Chris Brislawn and I. G. Rosen. Wavelet based approximation in the optimal control of distributed parameter systems. Numer. Funct. Anal. Optim., 12(1-2):33-77, 1991.

[10] X. X. Chen and Y. Y. Chen. Self-lifting scheme: new approach for generating and factoring wavelet filter bank. IET Signal Process., 2(4):405-414, 2008.

[11] Ingrid Daubechies and Wim Sweldens. Factoring wavelet transforms into lifting steps. J. Fourier Anal. Appl., 4(3):247-269, 1998.

[12] Yumin He, Xuefeng Chen, Jiawei Xiang, and Zhengjia He. Multiresolution analysis for finite element method using interpolating wavelet and lifting scheme. Comm. Numer. Methods Engrg., 24(11):1045-1066, 2008.

[13] Kenkichi Iwasawa. On some types of topological groups. Ann. of Math. (2), 50:507-558, 1949.

[14] A. Jensen and A. la Cour-Harbo. Ripples in mathematics. Springer-Verlag, Berlin, 2001. The discrete wavelet transform.

[15] Palle E. T. Jorgensen and Myung-Sin Song. Analysis of fractals, image compression, entropy encoding, Karhunen-Loève transforms. Acta Appl. Math., 108(3):489-508, 2009.

[16] Palle E. T. Jorgensen and Myung-Sin Song. Matrix Factorization and Lifting. Sampling Theory in Signal and Image Processing., 9:167-197, 2010.

[17] Wayne M. Lawton. Conjugate quadrature filters. In Advances in wavelets (Hong Kong, 1997), pages 103-119. Springer, Singapore, 1999.

[18] Wayne Lawton. Infinite convolution products and refinable distributions on Lie groups. Trans. Amer. Math. Soc., 352(6):2913-2936, 2000. 
[19] Wayne Lawton. Global analysis of wavelet methods for Euler's equation. Mat. Model., 14(5):75-88, 2002. Second International Conference OFEA'2001 "Optimization of Finite Element Approximation, Splines and Wavelets" (Russian) (St. Petersburg, 2001).

[20] Wayne M. Lawton. Hermite interpolation in loop groups and conjugate quadrature filter approximation. Acta Appl. Math., 84(3):315-349, 2004.

[21] B. W. K. Ling, C. Y. F. Ho, and J. D. Reiss. Control of sigma delta modulators via fuzzy impulsive approach. In Control of chaos in nonlinear circuits and systems, volume 64 of World Sci. Ser. Nonlinear Sci. Ser. A Monogr. Treatises, pages 245-270. World Sci. Publ., Hackensack, NJ, 2009.

[22] M Lammers, A. M. Powell, and Özgür Yılmaz. Alternative dual frames for digital-to-analog conversion in sigma-delta quantization. Adv. Comput. Math., 32(1):73-102, 2010.

[23] Peng-Lang Shui, Zheng Bao, and Yuan Yan Tang. Three-band biorthogonal interpolating complex wavelets with stopband suppression via lifting scheme. IEEE Trans. Signal Process., 51(5):1293-1305, 2003.

[24] Myung-Sin Song. Wavelet image compression. In Operator theory, operator algebras, and applications, volume 414 of Contemp. Math., pages 41-73. Amer. Math. Soc., Providence, RI, 2006.

[25] Wim Sweldens and Dirk Roose. Shape from shading using parallel multigrid relaxation. In Multigrid methods, III (Bonn, 1990), volume 98 of Internat. Ser. Numer. Math., pages 353-364. Birkhäuser, Basel, 1991.

[26] Wim Sweldens. The lifting scheme: a custom-design construction of biorthogonal wavelets. Appl. Comput. Harmon. Anal., 3(2):186-200, 1996.

[27] Wim Sweldens. The lifting scheme: a construction of second generation wavelets. SIAM J. Math. Anal., 29(2):511-546 (electronic), 1998.

[28] Walnut, D. F., An Introduction to Wavelet Analysis, (Birkhäuser, Boston, 2002). 